\title{
Ethanol Production in Yeasts Isolated from Fermented Kitchen Waste
}

\author{
S. WONG ${ }^{1}$, S. K. WONG ${ }^{1 *}$ AND J. S. BUJANG ${ }^{1}$
}

\begin{abstract}
Microbial ethanol is a potential substitute for the non-renewable fossil fuel which is depleting. Yeasts have been long and extensively studied for ethanol production. The objectives of this study were to isolate yeasts from fermented kitchen waste and to determine their ethanol production performances. A number of fifteen yeasts were isolated from fermented kitchen waste. The yeasts were then grouped based on their ability to ferment different types of sugars. Three yeast isolates were selected for the analysis of ethanol production. Fermentation was carried out for $72 \mathrm{~h}$ in yeast extract peptone dextrose broth containing $18 \%$ glucose. Fourier transform infrared attenuated total reflection spectroscopy was used to monitor the ethanol production and glucose utilization. Isolate Y4 achieved the highest ethanol production at the level of 16\%, while Y 6 and Y8 demonstrated $12 \%$ and $11 \%$ ethanol yields, respectively. The isolates Y4, Y6 and Y8 were identified using universal fungal primers ITS1 and ITS4. The yeast isolates were closest to Saccharomyces cerevisiae (76\%), Paracoccidioides brasiliensis (56\%) and Saccharomyces boulardii (64\%), respectively. This study showed that fermented kitchen waste could serve as a good source of yeasts for ethanol production.
\end{abstract}

Key words: Yeast; ethanol; fermentation; kitchen waste; FTIR-ATR; Saccharomyces cerevisiae; Paracoccidioides brasiliensis; Saccharomyces boulardii

Microbial ethanol is gaining worldwide acceptance to overcome problems associated with the depletion of fossil fuels and environmental pollution. The non-renewable energy sources have been depleting and it takes a few hundred million years for natural processes to recreate them. Therefore, the primary benefit of switching fuel source to microbial ethanol is that the biomass is renewable, and can potentially provide a sustainable supply over a long term. In addition, many estimate that the production and use of bioethanol could cause a reduction in net greenhouse gas emissions (Mabee \& Saddler 2010).

Ethanol production is usually done by chemical synthesis of hydrocarbons. In recent years, there is a global emphasis in ethanol production by fermentation process. Increased yield of ethanol production by microbial fermentation depends on the use of ideal microbial strain, appropriate fermentation substrate and suitable process technology. An ideal micro-organism used for ethanol production must have rapid fermentative potential, improved flocculating ability, appreciable osmotolerance, enhanced ethanol tolerance and good thermotolerance (Brooks 2008; Stewart et al. 1982; Yan \& Tanaka 2006). To date, there is no microbial strain which meet these qualities (Brooks 2008).

Brooks (2008) reported various strains of indigenous yeasts capable of producing ethanol had been isolated from different local sources such as molasses (Yan \& Tanaka 2006), sugar

\footnotetext{
${ }^{1}$ Department of Animal Science and Fishery, Faculty of Agriculture and Food Sciences, Universiti Putra Malaysia Bintulu Sarawak Campus

*Corresponding author (e-mail: wongsk@btu.upm.edu.my)
} 
mill effluents, local fermented foods, and fermented pineapple juice and lignocellulotic agroindustrial residues (Okur \& Saracçoĝlu 2006). In most of these studies, the preferred candidate for industrial production of ethanol has been Saccharomyces cerevisiae due to its ability to produce high concentration of ethanol and the ethanol is not contaminated by other products from the substrate (Yan \& Tanaka 2006).

Thus the aims of this study were to isolate indigenous yeast strains from the fermented kitchen waste and to evaluate their potential in ethanol production.

\section{MATERIALS AND METHODS}

Fermentation mixture was prepared by mixing kitchen waste (vegetables and fruits) and brown sugar and water at a weight ratio of $3: 1: 10$. Fermentation was carried out in a closed container at room temperature $\left(26^{\circ} \mathrm{C}-28^{\circ} \mathrm{C}\right)$ under non-sterile condition. The seven-dayold product was subjected to 10 -fold serial dilution then it was spread onto potato dextrose agar (PDA) containing $0.1 \%$ chloramphenicol. The PDA plates were incubated at $30^{\circ} \mathrm{C}$ for $24 \mathrm{~h}-48 \mathrm{~h}$. The yeast isolates were characterized by their ability to ferment sugars in broth containing $1 \%$ yeast extract and $2 \%$ sugars (glucose, lactose, galactose, fructose, xylose, maltose or sucrose).

Ethanol fermentation was carried out in conical flask at $26^{\circ} \mathrm{C}-28^{\circ} \mathrm{C}$ for $72 \mathrm{~h}$ in yeast extract peptone dextrose broth (1\% yeast extract, $2 \%$ peptone, $18 \%$ glucose) under sterile condition. Ethanol production and glucose utilization were monitored using Fourier transform infrared attenuated total reflection (FTIR-ATR) spectroscopy. The levels of ethanol and glucose in the samples were estimated based on the selected absorption wavenumber and absorbance value corresponding to those of the chemical standards. The isolates were identified through molecular approach based on the internal transcribed spacer (ITS) sequence. The DNA of the yeasts was extracted by boiling in $2 \%$ SDS followed by ethanol precipitation. The ITS region was amplified using universal primers ITS1 and ITS4 (White et al. 1990). Nucleotide sequences of the PCR products were analyzed by automated DNA sequencing by First Base Laboratories.

\section{RESULTS AND DISCUSSION}

Fifteen yeasts were isolated from fermented kitchen waste. They were grouped into six groups based on their sugar fermentation profiles (Table 1). Group 6 was the only group which did not ferment glucose and any other tested sugars. The Y7, Y9, Y10 and Y14 were therefore regarded as yeast-like microorganisms based on their colony morphologies and they were excluded from the fermentation test where glucose was used as carbon source. Primary fermentation analysis on the yeast isolates from the other 5 groups detected different levels of ethanol. Yeasts from groups 1, 2 and 3 produced sustaintial amount of ethanol as shown by the FTIR spectrums. However, there was only $<10 \%(\mathrm{v} / \mathrm{v})$ of ethanol observed in the culture media of yeast isolates in Groups 4 and 5 (data not shown).

Subsequent time course studies of ethanol production were performed on isolates Y4, Y6 and Y8, respectively representing group 1, 2 and 3. It was observed that ethanol production peaked after $36 \mathrm{~h}$ of fermentation. Y4 recorded the highest ethanol production as high as $16 \%$ followed by $12 \%$ and $11 \%$ in Y6 and Y8, respectively (Figure 1). Elevated level of ethanol in Y4 could be explained by efficient glucose utilization to below 1\% after $36 \mathrm{~h}$ fermentation (Figure 1). In contrast, glucose levels in Y6 and Y8 were 7\% and 5\%, respectively at $36 \mathrm{~h}$ and gradually decreased to $3 \%$ at 72 h. Ethanol has been reported to show inhibitory effects on yeast fermentation through 
Table 1. Sugar fermentation test of the yeasts.

\begin{tabular}{c|cccccccc}
\hline \multirow{2}{*}{ Yeasts } & \multicolumn{7}{|c}{ Sugars } & Grouping \\
\cline { 2 - 9 } & Glu & Mal & Lac & Gal & Xyl & Suc & Fru & 4 \\
\hline Y1 & + & - & - & + & - & + & + & 4 \\
Y2 & + & - & - & + & - & + & + & 4 \\
Y3 & + & - & - & + & - & + & + & 4 \\
\hline Y4 & + & + & - & + & - & + & + & 1 \\
\hline Y5 & + & - & - & - & - & - & - & 5 \\
\hline Y6 & + & + & - & - & - & + & + & 2 \\
\hline Y7 & - & - & - & - & - & - & - & 6 \\
\hline Y8 & + & - & - & - & - & - & + & 3 \\
\hline Y9 & - & - & - & - & - & - & - & 6 \\
Y10 & - & - & - & - & - & - & - & 6 \\
Y11 & + & - & - & - & - & - & - & 5 \\
Y12 & + & - & - & + & - & + & + & 4 \\
Y13 & + & - & - & - & - & - & + & 3 \\
Y14 & - & - & - & - & - & - & - & 6 \\
Y15 & + & - & - & - & - & - & - & 5 \\
\hline
\end{tabular}

Note: + : Positive fermentation; - : Negative fermentation; Glu: Glucose; Mal: Maltose; Lac: Lactose; Gal: Galactose; Xyl: Xylose; Suc: Sucrose; and Fru: Fructose.

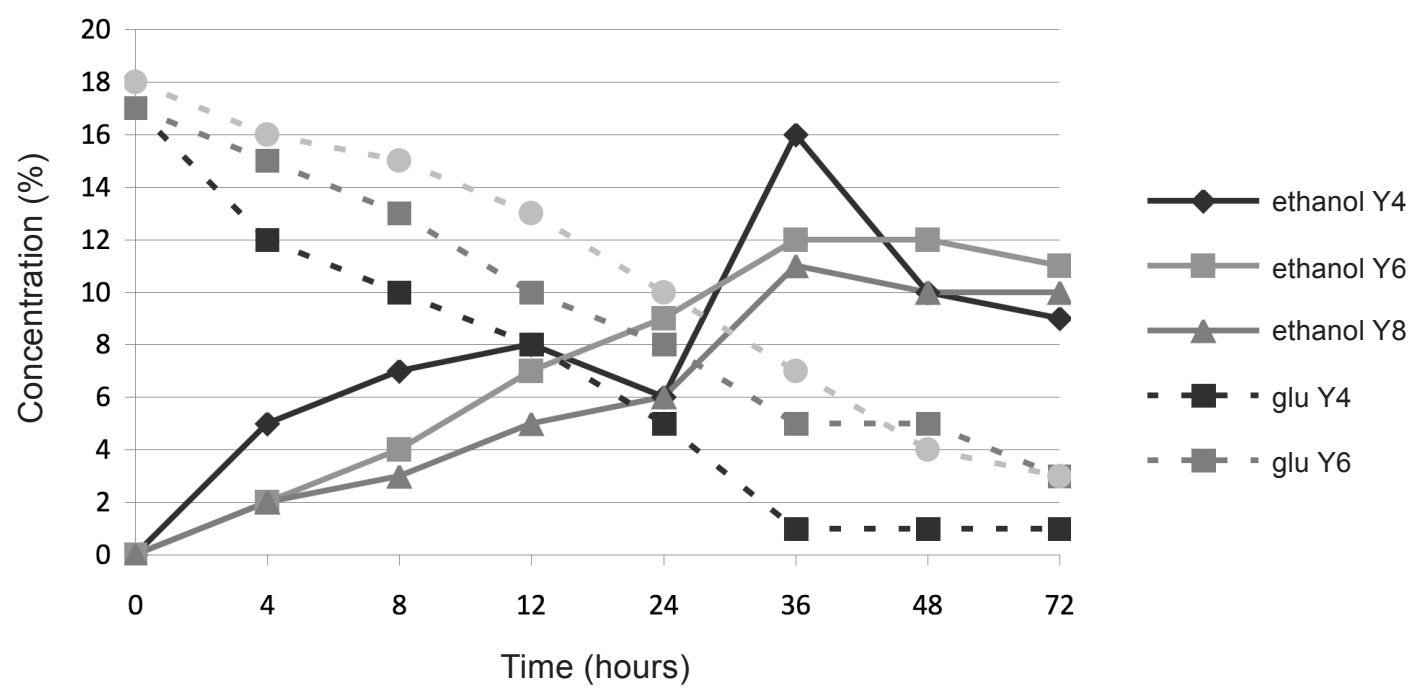

Figure 1. Time course study of ethanol fermentation in yeasts. 
the alteration of membrane permeability and disruption of its function (Dombek \& Ingram 1987). These lead to an increase in hydrogen ion influx which causes the decline in transport rates and eventually, there was no further glucose uptake for ethanol production.

FTIR spectroscopy offers rapid and convenient means in estimating the amounts of ethanol and glucose in fermentation mixture. There was no requirement for pre-analysis sample preparation and separation. The absorptions around $3000 \mathrm{~cm}^{-1}$ (likely a C-H stretch) (Coates 2000) and $1000 \mathrm{~cm}^{-1}$ (likely a C-O region) (Petibois et al. 1999) were used to quantitate the levels of ethanol and glucose respectively (Figure 2). These 2 wavenumbers were found to exhibit positive linear relationship towards different concentrations of ethanol $(0 \%-35 \%)$ and glucose $(0 \%-18 \%)$ standard at respective $\mathrm{R}^{2}$ values of 0.915 and 0.997 .

Isolates Y4, Y6 and Y8 generated PCR products at different sizes at 620 b.p., 500 b.p. and 520 b.p., respectively (Figure 3). ITS has been regarded as highly variable region for fungi identification. However, in this study, attempts to identify the yeasts isolates based on the ITS sequences were not conclusive due to low similarity $(<90 \%)$ between the sample sequences and the fungal DNA database. Isolates Y4, Y6 and Y8 were

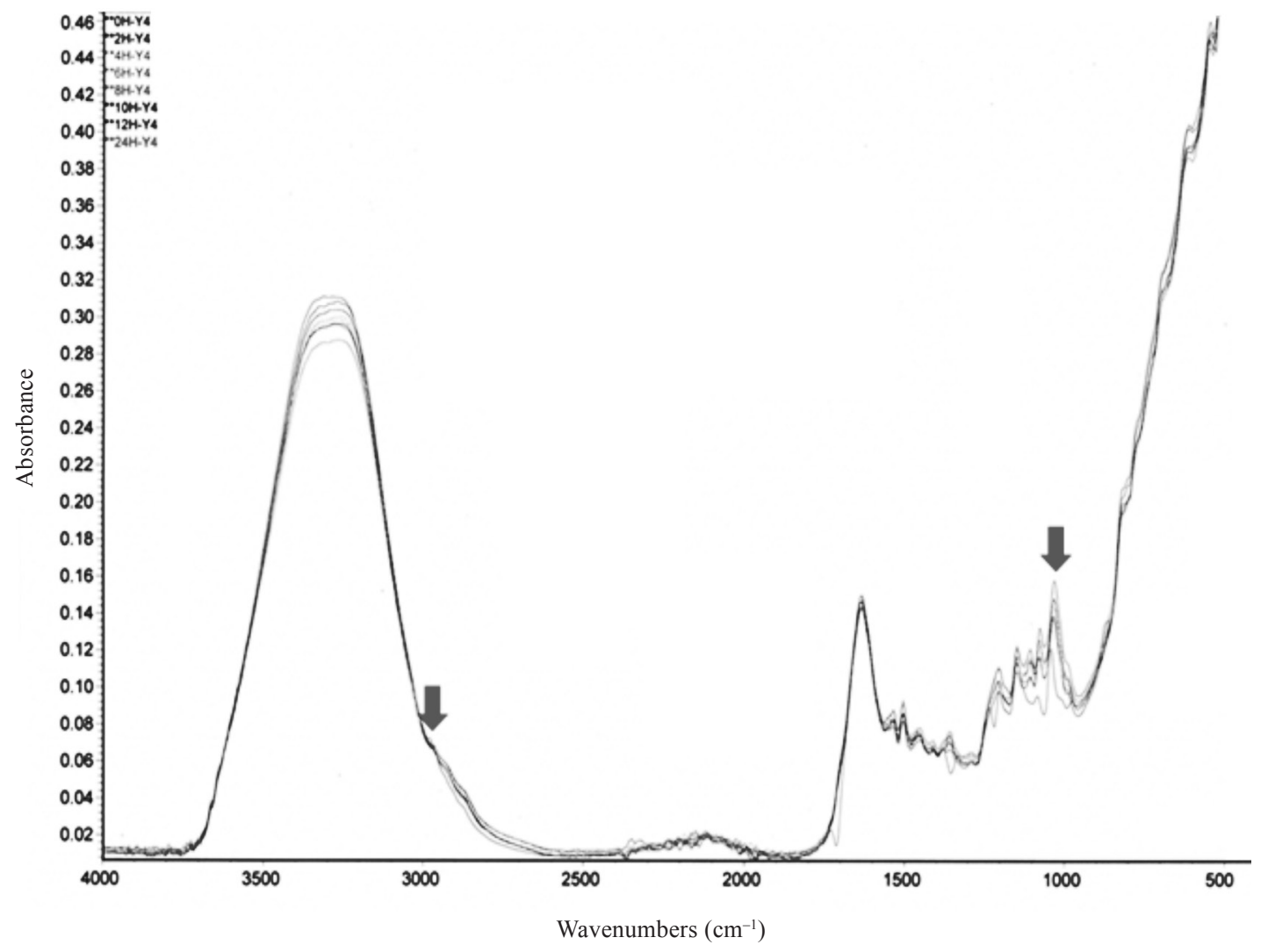

Figure 2. FTIR spectrum of yeast fermentation mixture: arrows indicate the absorption peaks used for quantitating ethanol $\left(3000 \mathrm{~cm}^{-1}\right)$ and glucose $\left(1000 \mathrm{~cm}^{-1}\right)$. 


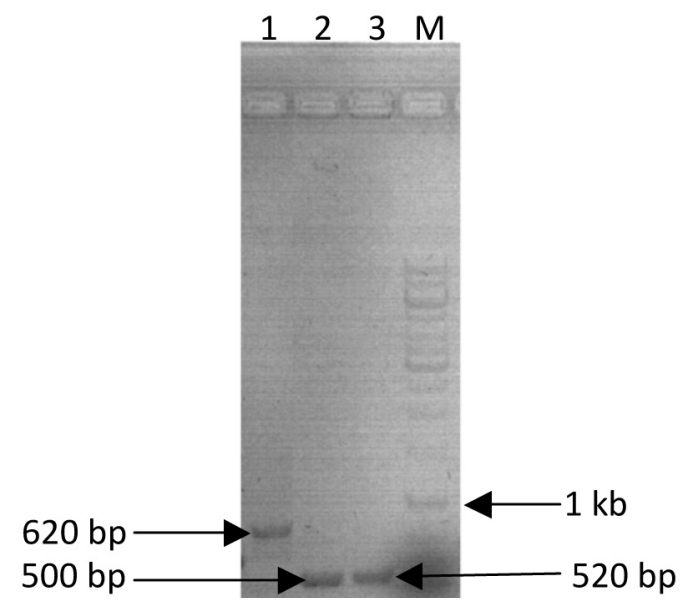

Figure 3. Amplified ITS regions of isolates $Y 4, Y 6$ and $Y 8$

Lane 1: Y4; lane 2: Y6; lane 3: Y8; M: VC $1 \mathrm{~kb}$ DNA ladder (Vivantis).

found closest to Saccharomyces cerevisiae (76\%), Paracoccidioides brasiliensis (56\%) and Saccharomyces boulardii (64\%) respectively based on the ITS sequences.

\section{CONCLUSION}

A number of three yeasts with promising ethanol production were isolated and partially identified from fermented kitchen waste. A rapid and simple FTIR based method has been developed to detect the ethanol and glucose levels during the fermentation process. The highest ethanol production was achieved in the yeast isolate $\mathrm{Y} 4$ which is believed to be closely related to Saccharomyces cerevisiae. Further analysis need to be carried out to verify the yeast species and its ethanol yield in a scale up of the fermentation process.

\section{ACKNOWLEDGEMENTS}

The authors thank Chiew C.H. for his help in conducting the experiments in this study.

Date of submission: January 2013

Date of acceptance: March 2013

\section{REFERENCES}

Brooks, AA 2008, 'Ethanol production potential of local yeast strains isolated from ripe banana peels'. African Journal of Biotechnology, vol. 7, pp. 3749-3752.

Coates, J 2000, 'Interpretation of infrared spectra, a practical approach', in Encyclopedia of Analytical Chemistry, ed RA Meyers, John Wiley \& Sons Ltd, Chichester, pp. 10815-10837.

Dombek, KM \& Ingram, LO 1987, 'Ethanol production during batch fermentation with Saccharomyces cerevisiae: changes in glycolytic enzymes and internal $\mathrm{pH}$, Applied and Environmental Microbiology, vol. 53, pp. 1286-1291.

Mabee, WE \& Saddler, JN 2010, 'Bioethanol from lignocellulosics: status and perspectives in Canada', Bioresource Technology, vol. 101, no. 13 , pp. $4806-4813$.

Okur, TM. \& Saracçoĝlu, NE 2006, 'Ethanol production from sunflower seed hull hydrolysate by Pichia stipitis under uncontrolled $\mathrm{pH}$ conditions in a bioreactor, Turkish Journal Engineering Enviromental Sciences, vol. 30, pp. 317-322.

Petibois, C, Rigalleau, V, Melin, AM, Perromat, A, Cazorla, G, Gin, H \& Déléris, G. 1999, 'Determination of glucose in dried serum samples by Fourier-transform infrared spectroscopy', Clinical Chemistry, vol. 45, pp. 1530-1535. 
Stewart, GG, Panchal, CJ \& Peacock, I 1982, 'Increased osmotolerance of genetically modified ethanol producing strains of Saccharromyces sp.. Biotechnology Letters, no. 4, pp. 639-644.

White, TJ, Bruns, TD, Lee, S \& Taylor, J 1990, 'Analysis of phylogenetic relationships by amplification and direct sequencing of ribosomal
RNA genes, in PCR Protocols: a Guide to Methods and Applications, eds MA Innis et al., Academic Press, New York, pp. 315-322.

Yan, L \& Tanaka, S 2006, 'Ethanol fermentation from biomass resources: current state and Prospects', Applied Microbiological Biotechnology, vol. 69, pp. 627-642. 